\title{
Estudo do ponto de corte da Escala de Quedas de Morse (MFS)
}

\author{
Study of the cut-off point of the Morse Fall Scale (MFS) \\ Estudio del punto de corte de la Escala de Caídas de Morse (MFS) \\ Maria José Martins da Costa-Dias*; Teresa Martins**; Fátima Araújo***
}

\section{Resumo}

Enquadramento: A Escala de Quedas de Morse (MFS) é uma escala de avaliação do risco de queda, desenhada para adultos e amplamente utilizada no nosso país. A escala deve ser calibrada para cada contexto para que as estratégias de prevenção sejam dirigidas aos doentes que estão em maior risco.

Objetivo: Analisar o ponto de corte da escala que melhor discrimine as pessoas sem risco das com risco de queda quando internadas em contexto hospitalar.

Metodologia: Foi utilizada para definir o melhor ponto de corte a receiver operator curve (ROC). A validade preditiva da MFS foi avaliada através da sensibilidade e especificidade, do valor preditivo positivo e negativo, da área abaixo da curva $(A U C)$ de $R O C$ e ainda através do Índice de Youden.

Resultados: O ponto de corte 45 foi identificado como o melhor ponto de corte, no qual 78\% dos participantes são identificados como verdadeiros positivos e $22 \%$ como falsos negativos e apresenta um $O R$ de 3,8 (IC95\% $=2,17-6,51)$.

Conclusão: A MFS revela ser um bom instrumento para identificar doentes com alto risco de queda, em contexto hospitalar, particularmente em internamentos médico-cirúrgicos, de cuidados continuados e paliativos.

Palavras-chave: acidentes por quedas; controle do risco; serviços hospitalares.

\begin{abstract}
Theoretical framework: The Morse Fall Scale (MFS) is a scale for assessing fall risk. It is designed for adults and widely used in Portugal. The scale should be calibrated for each particular setting so that fall prevention strategies are targeted to patients most at risk.

Objective: To analyse the cut-off point of the scale that best distinguishes between people with and without fall risk in a hospital inpatient setting.

Methodology: The receiver operator curve (ROC) was used to determine the optimal cut-off point. The predictive validity of the MFS was assessed through sensitivity and specificity, positive and negative predictive values, the ROC area under the curve (AUC) and also the Youden Index.

Results: The cut-off point of 45 was identified as the optimal cut-off value, where $78 \%$ of the participants were identified as true positives and $22 \%$ as false negatives. It presented an $O R$ of $3.8(95 \% C I=2.17-6.51)$.

Conclusion: The MFS is a good instrument for identifying patients with high fall risk in hospital settings, particularly in medical and surgical, long-term and palliative care inpatient wards.
\end{abstract}

Keywords: accidental falls; risk management; hospital services.

* Enfermeira Especialista em Saúde Infantil e Pediátrica. Aluna do Doutoramento em Enfermagem do Instituto de Ciências da Saúde da Universidade Católica Portuguesa. Morada: Avenida Lusíada, 100,1500-650 Lisboa, Portugal [cdias@ hospitaldaluz.pt].

* Professora Coordenadora, Escola Superior de Enfermagem do Porto. Doutora em Ciências da Educação, 4200-072 Porto, Portugal[teresam@esenf.pt].

*** Professora Adjunta. Doutora em Ciências da Educação. Escola Superior de Enfermagem do Porto, 4200-072 Porto, Portugal[araujo@esenf.pt].

\section{Resumen}

Marco contextual: la Escala de Caídas de Morse (MFS) es una escala para evaluar el riesgo de caídas, diseñada para adultos y ampliamente utilizada en Portugal. La escala debe ser calibrada para cada contexto, con el fin de que las estrategias de prevención se dirijan a los pacientes que están en mayor riesgo. Objetivo: identificar el punto de corte de la escala que mejor discrimine a las personas que no tienen riesgo de caída de las que sí lo tienen cuando están internas en un contexto hospitalario.

Metodología: para determinar el mejor punto de corte, se utilizó la curva del receptor operador (ROC). La validez predictiva de la MFS se evaluó por medio de la sensibilidad y la especificidad, del valor predictivo positivo y negativo, del área bajo la curva (AUC) de ROC y también a través del Índice de Youden.

Resultados: el punto de corte de 45 fue identificado como el mejor punto de corte, en el que se identifica el $78 \%$ de los participantes como verdaderos positivos y el $22 \%$ como falsos negativos, y tiene un OR de 3,8 (IC95\%=2,17-6,51).

Conclusión: la MFS es un buen instrumento para identificar pacientes con alto riesgo de caídas en el ámbito hospitalario, sobre todo en pacientes hospitalizados en servicios médicos y quirúrgicos, así como de cuidados continuos y paliativos.

Palabras clave: accidentes por caídas; control de riesgo; servicios hospitalarios.

Recebido para publicação em: 10.04 .13

Aceite para publicação em: 18.01 .14 


\section{Introdução}

As escalas de avaliação do risco de queda são ferramentas que atribuem um valor numérico a diversos fatores de risco. Estes são somados de forma a predizerem se o doente tem ou não risco de queda. A avaliação da validade preditiva das escalas (Morse, 2009) permite identificar se as mesmas servem o seu propósito, isto é, se descriminam suficientemente bem entre potenciais doentes com risco de queda e doentes sem risco, numa população específica (Oliver, Healey, \& Haines, 2010). A Morse Fall Scale (MFS) é uma das escalas mais utilizada, tendo em consideração o seu extenso desenvolvimento e estudos comparativos em populações de doentes internados em diferentes hospitais (Schwendimann, De Geest, \& Milisen, 2006).

Uma escala de avaliação do risco de queda, aliás como qualquer outro instrumento de avaliação necessita de ser submetido a um processo de adaptação cultural e linguística e validado para a língua portuguesa, se o desejamos utilizar nos nossos contextos (Almeida, Abreu, \& Mendes, 2010). Idealmente as escalas devem ser calibradas para cada unidade, para que as estratégias de prevenção sejam dirigidas aos doentes que estão mais em risco, ou seja, os pontos de corte ou os valores que fazem a separação entre um doente sem e com risco de queda, podem ser diferentes dependendo se está a ser utilizada a escala num hospital de doentes agudos ou num hospital de doentes crónicos. Mesmo dentro da mesma organização a escala pode ter pontos de corte diferentes (Morse, 2009). O risco de queda varia em função do tipo de doente, assim como em diferentes momentos do dia e nas diferentes situações do doente (Morse, 2009). Os pontos de corte definidos pela autora da escala situam-se no intervalo entre $25 \mathrm{e}$ 55 pontos, sugerindo que o ponto de corte para alto risco de queda num hospital de doentes agudos, ou numa unidade de doentes cirúrgicos onde poucos doentes terão um risco de queda alto, seja colocado no score 25. Pelo contrário nos hospitais ou nas unidades destinadas a doentes crónicos ou unidade de doentes com patologia cerebrovascular, a autora sugere que o score de alto risco seja aferido para o score 45. Morse (2009) alerta também que o ponto de corte da escala não deve situar-se acima dos 55 pontos. Este valor foi identificado por Schwendimann et al. (2006) num estudo de coorte prospetivo efetuado num hospital de doentes agudos em dois internamentos de medicina, como o ponto de corte mais ajustado aos doentes internados nos serviços referidos.

O melhor método para definir o ponto de corte de acordo com Morse (2009) é avaliar o risco a todos os doentes internados num determinado serviço e analisar a distribuição do risco, obtendo os valores da sensibilidade (verdadeiros positivos, ou a proporção de participantes com queda corretamente classificados com alto risco) e especificidade (verdadeiros negativos, ou a proporção de participantes sem quedas corretamente classificados sem risco ou com baixo risco) da escala, assim como os valores preditivos positivos (probabilidade de uma pessoa com avaliação de risco cair) e negativos (probabilidade de uma pessoa com avaliação sem risco não cair).

O objetivo deste estudo foi analisar, para os serviços de internamento de adultos de um hospital da zona da grande Lisboa de doentes agudos, o ponto de corte da MFS, mais ajustado à sua população.

\section{Fundamentação Teórica}

O acidente queda do doente é definido por Lamb, Jorstad-Stein, Hauer, e Becker, (2005) como um incidente em que a pessoa súbita e involuntariamente desce até ao nível do chão ou a um nível mais baixo. As quedas estão na base do maior número de danos não fatais nos hospitais de doentes agudos (Perell, Nelson, Goldman, Prieto-Lewis, \& Rubenstein, 2001), afetando maioritariamente os idosos (Oliver, 2007), sendo um evento adverso que é prevenível. Escalas simples de avaliação de risco podem predizer até $70 \%$ das quedas (Oliver, Daly, Martin, \& McMurdo, 2004). A primeira etapa de qualquer programa de intervenção de quedas é a avaliação do risco, o enfermeiro deve utilizar instrumentos de avaliação adequados para identificar riscos reais e potenciais dos clientes que cuida, competência definida a nível da gestão de cuidados no domínio do ambiente seguro, pela Ordem dos Enfermeiros (2004). A Escala de Quedas de Morse (MFS) é uma das escalas mais divulgada e utilizada a nível nacional (Soares \& Almeida, 2008), tendo sido demonstrada a sua validade preditiva, bem como a sua fiabilidade entre avaliadores (Morse, 2006; Morse, 2009).

A MFS é descrita pela sua autora como sendo um método simples e rápido de avaliar a probabilidade de 
um doente cair, tendo duas décadas de investigação. A escala foi desenvolvida em 1985 no Canadá, na Universidade de Alberta, por Janice Morse, para identificar os indivíduos com risco de quedas (Morse, Morse, \& Tylko, 1989).

A escala foi desenvolvida com base num estudo de 100 doentes que caíram e de 100 doentes que não caíram, selecionados de forma randomizada.

A escala integra seis itens de avaliação e que são: (1) história anterior de queda; (2) existência de um diagnóstico secundário; (3) apoio para caminhar; (4) terapia intravenosa; (5) postura no andar e na transferência; (6) estado mental.

Os itens 1,2,4e6 são medidos numa escala dicotómica Não/Sim em que "Não" toma sempre o valor zero e "Sim" o valor 15 (nos itens 2 e 6), 20 (no item 4) ou 25 (no item 1). 0 item 3 tem como respostas possíveis "Nenhuma/ajuda de enfermeiro/acamado/cadeiras de rodas" (0), "Muletas/canadianas/bengala/andarilho" (15) ou "Apoia-se no mobiliário para andar" (30). Por fim, o item 5 tem como respostas possíveis "Normal/acamado/imóvel" (0), "Debilitado" (10) e "Dependente de ajuda" (20).

A pontuação total da escala varia entre 0 e 125 e os indivíduos são descriminados em função do risco apresentado como: sem risco (0-24), baixo risco (25$50)$ e alto risco $(\geq 51)$.

No estudo original a sensibilidade da escala foi de $78 \%$, o valor preditivo positivo foi de $10,3 \%$ e o valor preditivo negativo de 99,2\% (Morse et al., 1989).

A escala foi testada dois anos após a sua construção, numa amostra de doentes idosos, em contexto hospitalar, tendo demonstrado especificidade de $51 \%$, sensibilidade de $72 \%$, valor preditivo positivo de $38 \%$, valor preditivo negativo de $81 \%$, e precisão de 57\% (Eagle, Salama, Whitman, \& Evans, 1999).

Em 2006 o ponto de corte da MFS foi estudado por Schwendimann et al., para uma população de 368 doentes, dos quais $60 \%$ eram do sexo feminino, com uma média de etária de 70 anos $(D P \pm 18,5)$, com internamentos superiores a 48 horas e com diferentes motivos médicos de internamento. Dos participantes deste estudo (47) tiveram 69 quedas. Os participantes com risco de queda variaram entre $89,4 \%$ para um ponto de corte 20 , até $20,7 \%$ para um ponto de corte de 70 pontos. De acordo com os diferentes pontos de corte deste estudo a sensibilidade da MFS variou entre $91,5 \%$ e $38,3 \%$. A especificidade variou entre $81,7 \%$ e $10,9 \%$. O valor preditivo positivo entre $12,5 \%$ a $22,5 \%$ e o valor preditivo negativo entre $90,2 \%$ a $95,7 \%$. Os falsos positivos ou os participantes com risco mas que não caíram variaram entre $87,5 \%$ para o ponto de corte 20 e 75,9\% para o ponto de corte de 60 .

Em 2011 foi criada a Versão Portuguesa da MFS e validada para a realidade Portuguesa numa amostra de 200 doentes, aguardando-se a sua publicação. Como resultado desta validação foi criada uma versão semântica e culturalmente equivalente à original, com uma boa fiabilidade e validade (relações significativas com a Escala de Coma de Glasgow e com o Índice de Barthel). A fiabilidade foi testada através do grau de concordância das pontuações fornecidas por três enfermeiros, independentemente. Este grau de concordância variou de 0,62 a 0,96 e o correspondente coeficiente de correlação interclasse foi de 0,84, associado a um intervalo de confiança de $[0,80 ; 0,88]$, pelo que existiu uma elevada concordância entre os avaliadores na utilização da escala.

Oliver (2007) refere que a avaliação da validade preditiva de uma escala tem como componentes a sensibilidade, a especificidade, o valor preditivo positivo e negativo e a precisão.

\section{Metodologia}

Através de um estudo do tipo caso-controlo, foram incluídos 100 registos de incidente de queda dos 134 notificados durante o período de 1 de janeiro a 31 de dezembro do ano de 2012, por cinco serviços de internamento de doentes adultos na área médicocirúrgica e de cuidados continuados e paliativos, identificados como A, B, C, D e E. No ano em estudo registou-se uma incidência de 2,4 quedas por 1000 camas/dia. Os casos correspondem a registos de pessoas que sofreram o incidente queda no período de internamento. Por cada caso foram selecionados, de forma aleatória, dois controlos emparelhados por idade e sexo entre os registos de indivíduos adultos internados no mesmo período e sem registo de quedas no internamento.

Os dados foram introduzidos em base de dados elaborada através do programa estatístico SPSS ${ }^{\circledR}$ Statistical Package for the Social Sciences versão 18.0 para o Windows ${ }^{\circledR}$. Efetuou-se uma análise univariada dos dados com recurso a medidas de tendência central e de dispersão, bem como análise bivariada com 
recurso a medidas de risco, nomeadamente o odds ratio $(\mathrm{OR})$, com um intervalo de confiança de $95 \%$.

As propriedades métricas da escala foram avaliadas através da sensibilidade e da especificidade da escala para vários pontos de corte possíveis, através de pares de sensibilidade e especificidade para cada ponto de corte. Para definir o melhor ponto de corte foi utilizada a receiver operator curve ( $\mathrm{ROC}$ ), avaliada a eficácia da MFS através da área abaixo da curva (AUC) ROC, da precisão (ACC) e ainda através do Índice de Youden com intervalo de confiança de $95 \%$. A AUC tem um intervalo entre 0,5 a 1,0, com os valores mais elevados a indicar maior ajuste. O índice de Youden (J) é igual à sensibilidade mais a especificidade menos um. $O J$ tem uma variação entre 0 e 1 , com os valores próximos de $1 \mathrm{a}$ indicar a precisão perfeita e o valor 0 a precisão obtida por mero acaso (Schisterman, Perkins, Liu, \& Bondell, 2005).

Foram observados todos os requisitos éticos de um trabalho desta natureza, tendo o estudo sido autorizado pela Comissão de Ética para a Saúde do hospital onde se realizou o estudo.

\section{Resultados}

Os meses de maio (11) e janeiro (11) foram os meses com ocorrência de mais quedas. Contrariamente dezembro (4), fevereiro (6), abril (6) e agosto (6) mostraram ser os meses com menor número de eventos de queda. A média de quedas por mês foi de $8(D P \pm 2,64)$, a moda foi de 6 e a mediana 9, com um valor mínimo mensal de ocorrência de quedas de $4 \mathrm{e}$ máximo de 13.
Registou-se um maior número de quedas em participantes do sexo masculino (56\%) que nos participantes do sexo feminino.

Através do Tabela 1 pode ser feita a leitura das características dos participantes. A média da idade dos participantes foi de 76 anos ( $D P \pm 11,13)$, a moda 75 anos, a mediana 77 anos e a idade mínima de 26 anos e a máxima de 94 anos. Dos participantes 34\% (103) têm uma idade compreendida entre 70 aos 79 anos. Nos casos a idade média foi de 76 anos (DP $\pm 11,1)$, a moda é 79 anos e a mediana 77 anos, a idade mínima de 28 anos e máxima de 92 anos. A maior parte das ocorrências de quedas foram no serviço B com 42\% (42) dos casos, correspondendo a um serviço de internamento de doentes com necessidade de convalescença e reabilitação, ou com doença crónica incurável, progressiva e avançada.

Os casos foram internados em 37\% (42) das situações por motivos médicos e em 26\% (26) por necessidade de cuidados paliativos. Nos controlos o motivo de internamento foi em 34,5\% (69) por motivos cirúrgicos e em 33,5\% (67) por motivos médicos.

A doença oncológica é diagnóstico principal em 27\% (27) dos casos e em 13\% (39) dos controlos.

Através da Tabela 2 verificou-se que até ao $6^{\circ}$ dia de internamento houve um registo de ocorrência de queda de $57 \%$ e até ao $15^{\circ}$ dia de internamento, $72 \%$ das pessoas. Mais de metade dos participantes têm o evento de queda até aos 4,5 dias após o internamento, sendo que o maior registo absoluto deste incidente (moda) acontece nas primeiras 24 horas. Ainda de realçar que $11,0 \%$ (11) caíram ao $27^{\circ}$ dia ou após esta data.

TABELA 1 - Características dos participantes ( $\mathrm{n}=300)$

\begin{tabular}{|c|c|c|c|c|c|}
\hline & \multirow{2}{*}{ Variáveis } & \multicolumn{2}{|c|}{ Casos } & \multicolumn{2}{|c|}{ Controlos } \\
\hline & & $N^{o}$ & $\%$ & $N .^{o}$ & $\%$ \\
\hline \multirow[t]{7}{*}{ Idade } & $\leq$ a 39 anos & 1 & 1,0 & 2 & 2,0 \\
\hline & De 40 a 49 anos & 2 & 2,0 & 4 & 2,0 \\
\hline & De 50 a 59 anos & 3 & 3,0 & 6 & 3,0 \\
\hline & De 60 a 69 anos & 21 & 21,0 & 40 & 20,0 \\
\hline & De 70 a 79 anos & 34 & 34,0 & 69 & 35,5 \\
\hline & De 80 a 89 anos & 32 & 32,0 & 64 & 32,0 \\
\hline & $\geq$ a 90 anos & 7 & 7,0 & 15 & 7,5 \\
\hline \multirow[t]{5}{*}{ Serviços } & A & 3 & 3,0 & 6 & 3,0 \\
\hline & B & 42 & 42,0 & 84 & 42,0 \\
\hline & C & 28 & 28,0 & 56 & 28,0 \\
\hline & $\mathrm{D}$ & 26 & 26,0 & 52 & 26,0 \\
\hline & $\mathrm{E}$ & 1 & 1,0 & 2 & 1,0 \\
\hline
\end{tabular}




\begin{tabular}{llcccc} 
Motivo de Internamento & 42 & 42,0 & 67 & 33,5 \\
& Médico & 19 & 19,0 & 69 & 34,5 \\
& Cirúrgico & 12 & 12,0 & 17 & 8,5 \\
& Cuidados Continuados & 26 & 26,0 & 41 & 20,5 \\
& Cuidados paliativos & 1 & 1,0 & 6 & 3,0 \\
& Outro motivo & 10 & 10,0 & 21 & 7,0 \\
Diagnóstico Principal & Doença Cardiovascular & 11 & 11,0 & 10 & 3,3 \\
& Doença Respiratória & 4 & 4,0 & 11 & 3,7 \\
& Doença Urológia & 18 & 18,0 & 17 & 5,7 \\
& Doença do SNC* & 4 & 4,0 & 21 & 7,0 \\
& Doença Osteoarticular & 27 & 27,0 & 39 & 13,0 \\
& Doença Oncológica & 10 & 10,0 & 30 & 10,0 \\
& Doença Gastrointestinal & 6 & 6,0 & 14 & 4,7 \\
& Doença Infecciosa & 2 & 2,0 & 1 & 0,3 \\
& Doença Psiquiátrica & 8 & 8,0 & 36 & 12,0 \\
\hline
\end{tabular}

*Doença Neurodegenerativa e Cerebrovascular;

+ Doença vascular, hepática, renal, endócrina, da parede abdominal, gravidez, cirurgia estética, hematoma subdural.

TABELA 2 - Dias de internamento em que ocorreram as quedas

\begin{tabular}{lccccccc}
\hline Dias de ocorrência da queda & $N^{{ }^{o}}$ & $\%$ & $M$ & Mo & Md & DP & Min-Máx \\
\hline & & & 10,3 & 1,0 & 4,5 & $\pm 15,2$ & $1-113$ \\
$\leq 2$ dias & 33 & 33,0 & & & & & \\
Entre 3 a 6 dias & 24 & 24,0 & & & & \\
Entre 7 a 10 dias & 15 & 15,0 & & & & \\
Entre 11 a 14 dias & 8 & 8,0 & & & & \\
Entre 15 a 18 dias & 3 & 3,0 & & & & \\
Entre 19 a 22 dias & 3 & 3,0 & & & & \\
Entre 23 a 26 dias & 3 & 3,0 & & & & \\
$\geq 27$ dias & 11 & 11,0 & & & & & \\
\hline
\end{tabular}

A avaliação do risco de queda efetuada através da MFS pode ser lida na Tabela 3 e informa a nível da sua primeira questão sobre história anterior de queda, que 31\% (31) dos casos tinham história anterior de queda nos últimos 3 meses que antecederam o internamento, ou a queda foi o motivo de admissão de urgência hospitalar, assim como em 17\% (34) dos controlos. Existe associação estatisticamente significativa entre ter quedas anteriores e o risco de queda $\left(X^{2}=74,456 ; g l=2 ; p=0,000\right)$, sendo que $94 \%$ (61) das pessoas com quedas anteriores tinham alto risco de queda. Do total dos casos 92\% (92) têm um diagnóstico secundário, assim como 80,5\% (161) dos controlos.

No que se refere à questão sobre necessidade de ajuda para caminhar, verificou-se que 42\% (42) dos casos podiam andar sem qualquer ajuda, ou são apenas assistidos por um enfermeiro, andavam de cadeira de rodas, ou permaneciam deitados e nunca saíam da cama, sendo esta avaliação também igual para 66,5\% (133) dos controlos. Ainda nesta questão em 37\% (37) dos casos e em 18,5\% (37) dos controlos, as pessoas deslocavam-se apoiando-se numa peça de mobiliário.
Por fim em 21\% (21) dos casos e em 15\% (30) dos controlos, os participantes usavam, canadianas de antebraço, bengala ou outro dispositivo de apoio à marcha.

Quanto à questão da existência de terapia intravenosa, constatou-se que 58\% (58) dos casos tinham acessos com perfusões em curso de forma permanente ou transitória, assim como 63\% (126) dos controlos.

Na questão postura do andar e na transferência, 38\% (38) dos casos tinham um andar debilitado, em que as pessoas andavam curvadas, mas eram capazes de levantar a cabeça enquanto andam, sem perder o equilíbrio. Os passos eram curtos e podiam arrastar os pés. Esta avaliação é também aplicada a $24,5 \%$ (49) dos controlos. Andar dependente de ajuda (com dificuldade em se levantar da cadeira, tenta pôr-se de pé, apoiar-se nos braços da cadeira ou balancearse), colocar a cabeça para baixo e olhar para o chão, ou andar, agarrado ao mobiliário, a uma pessoa, ou a ajudas técnicas, ou mesmo não conseguir andar sem ajuda, foram identificados 34\% (34) dos casos e $22,5 \%$ dos controlos. Verificou-se ainda que 28\% (28) dos casos têm um andar normal, que se caracterizava 
por a pessoa andar com a cabeça levantada, baloiçar os braços livremente dos lados do corpo e dar passos largos sem hesitação. Esta avaliação foi também comum a 53\% (106) dos controlos.

A última questão avalia o estado mental das pessoas, sendo que 50\% (50) dos casos estavam conscientes das suas capacidades, avaliação feita com base na autoavaliação que a própria pessoa faz da sua capacidade e $50 \%$ (50) das pessoas sobrevalorizavam as suas capacidades e esqueciam-se das suas limitações. Quanto aos controlos 65\% (130) enquadram-se na primeira avaliação referida.

Verificou-se que as pessoas com história anterior de quedas $(O R=2,19 ; I C=1,25-3,83)$, que utilizam apoios de marcha para caminharem $(O R=2,22$; $I C=1,15-4,28)$ e que se esquecem das suas limitações $(O R=1,86 ; \quad I C=1,14-3,03)$ apresentam uma probabilidade 2 vezes superior de cair. As pessoas que têm um diagnóstico secundário $(O R=2,79 ; I C=1,25$ $6,22)$, que se apoiam no mobiliário para andarem $(O R=3,17 ; C C=1,79-5,63)$, que têm uma postura no andar e na transferência debilitada $(O R=2,94$; $I C=1,62-5,32)$, ou dependente de ajuda $(O R=2,86$; $I C=1,56-5,26)$, ou comprometida $(O R=2,70$; $I C=1,73-4,86)$, têm uma probabilidade 3 vezes superior de cair.

Da avaliação dos seis itens da MFS, resulta o nível de risco dos participantes, em que 65\% (65) dos casos mostraram ter alto risco e 41,5\% (83) dos controlos baixo risco de queda. O score da escala variou entre o mínimo 0 e o máximo de 125, com uma média de $54(D P \pm 29,04)$, indicativo de alto risco de queda. Metade dos participantes apresentou um score de risco superior a 50 .

TABELA 3 - Avaliação do risco de queda de casos e controlos e cálculo do odds ratio

\begin{tabular}{|c|c|c|c|c|c|}
\hline \multirow{2}{*}{ Avaliação do Risco de Queda } & \multicolumn{2}{|c|}{ Casos } & \multicolumn{2}{|c|}{ Controlos } & \multirow{2}{*}{$O R$} \\
\hline & $N .^{o}$ & $\%$ & $N .^{o}$ & $\%$ & \\
\hline \multicolumn{6}{|l|}{ História de quedas } \\
\hline Não & 69 & 69,0 & 166 & 83,0 & \\
\hline Sim & 31 & 31,0 & 34 & 17,0 & $2,19[1,25 ; 3,83]$ \\
\hline \multicolumn{6}{|l|}{ Diagnóstico secundário } \\
\hline Não & 8 & 8,0 & 39 & 19,5 & \\
\hline Sim & 92 & 92,0 & 161 & 80,5 & $2,79[1,25 ; 6,22]$ \\
\hline \multicolumn{6}{|l|}{ Ajuda para caminhar } \\
\hline \multicolumn{6}{|l|}{ Nenhuma/ajuda de } \\
\hline enfermeiro/acamado/cadeira de rodas* & 42 & 42,0 & 133 & 66,5 & - \\
\hline Muletas/canadianas/bengala/andarilho & 21 & 21,0 & 30 & 15,0 & $2,22[1,15 ; 4,28]$ \\
\hline Apoia-se no mobiliário para andar & 37 & 37,0 & 37 & 18,5 & $3,17[1,79 ; 5,63]$ \\
\hline \multicolumn{6}{|l|}{ Terapia intravenosa } \\
\hline Sem terapia intravenosa & 42 & 42,0 & 74 & 37,0 & \\
\hline Com terapia intravenosa & 58 & 58,0 & 126 & 63,0 & n.s. \\
\hline \multicolumn{6}{|l|}{ Postura no andar e na transferência } \\
\hline Normal/acamado/imóvel* & 28 & 28,0 & 106 & 53,0 & - \\
\hline Debilitado & 38 & 38,0 & 49 & 24,5 & $2,94[1,62 ; 5,32]$ \\
\hline Dependente de ajuda & 34 & 34,0 & 45 & 22,5 & $2,86[1,56 ; 5,26]$ \\
\hline \multicolumn{6}{|l|}{ Estado mental } \\
\hline Consciente das suas capacidades & 50 & 50,0 & 130 & 65,0 & \\
\hline Esquece-se das suas limitações & 50 & 50,0 & 70 & 35,0 & $1,86[1,14 ; 3,03]$ \\
\hline
\end{tabular}

Legenda:*classe de referência n.s. = não significativo

Tendo por base de referência a classe sem risco de queda, identificou-se que os participantes com baixo risco de queda apresentavam 3 vezes mais probabilidade de cair. Todavia, os participantes identificados com alto risco de queda mostraram ter 7 vezes mais probabilidade de sofrer um evento de queda durante o período de internamento (Tabela 4). 
TABELA 4 - Nível de risco dos casos e controlos e cálculo do odds ratio

\begin{tabular}{lccccc}
\hline & \multicolumn{3}{c}{ Casos } & \multicolumn{3}{c}{ Controlos } & \multirow{2}{*}{ OR } \\
\cline { 2 - 5 } Nivel de risco & $N^{o}$ & $\%$ & $N^{o}$ & $\%$ & - \\
\hline Sem risco* & 5 & 5,0 & 42 & 21,0 & $3,04[1,09 ; 8,39]$ \\
Baixo risco & 30 & 30,0 & 83 & 41,5 & $7,28[2,72 ; 19,49]$ \\
Alto risco & 65 & 65,0 & 75 & 37,5 & \\
\hline
\end{tabular}

Legenda: *Classe de referência

Com base nestes indicadores a sensibilidade da escala é de $65 \%$, ou seja permite identificar $65 \%$ das pessoas em risco que caíram. A sua especificidade é de $63 \%$ ao permitir identificar $63 \%$ das pessoas que não caíram e não tinham risco de queda. Têm um valor preditivo positivo (VPP) de $46 \%$, que permiteidentificarque $46 \%$ das pessoas com avaliação de risco têm probabilidade de cair e tem um valor preditivo negativo (VPN) de $78 \%$, que permite identificar que $22 \%$ das pessoas sem risco têm também probabilidade de cair.

Testou-se a MFS, através da sensibilidade e da especificidade da escala para vários pontos de corte possíveis, através de pares de sensibilidade e especificidade para cada ponto de corte. De acordo com os diversos pontos de corte a sensibilidade variou entre 96\% e 47\%, a especificidade variou entre $75 \%$ e $11 \%$, o VPP entre $35 \%$ e $48 \%$ e o VPN entre $84 \%$ e $74 \%$. As percentagens de falsos positivos (participantes com risco e sem queda) variaram entre $89,5 \%$ (ponto de corte: 20 ) e $25,5 \%$ (ponto de corte: 70). As percentagens de falsos negativos (participantes sem risco e com queda) variaram entre 5\% (ponto de corte: 20) e 53\% (ponto de corte: 70). A ACC, ou a precisão da escala, variou entre 39\% e 65\%. A AUC ou a área abaixo da curva ROC variou entre 0,533 e 0,608 e o J entre 0,070 e 0,300 (Tabela 5).

TABELA 5 - Validade preditiva dos pontos de corte da MFS $(n=300)$

\begin{tabular}{lccccccccc}
\hline Pontos de corte & 20 & 25 & 35 & 45 & 50 & 55 & 60 & 65 & 70 \\
\hline Sensibilidade & $96 \%$ & $95 \%$ & $90 \%$ & $78 \%$ & $73 \%$ & $65 \%$ & $56 \%$ & $51 \%$ & $47 \%$ \\
Especificidade & $11 \%$ & $20 \%$ & $29 \%$ & $52 \%$ & $56 \%$ & $63 \%$ & $67 \%$ & $71 \%$ & $75 \%$ \\
$V P P^{i}$ & $35 \%$ & $37 \%$ & $39 \%$ & $45 \%$ & $45 \%$ & $46 \%$ & $46 \%$ & $47 \%$ & $48 \%$ \\
$V P N^{b}$ & $84 \%$ & $89 \%$ & $85 \%$ & $82 \%$ & $80 \%$ & $78 \%$ & $75 \%$ & $74 \%$ & $74 \%$ \\
$A C C^{c}$ & $39 \%$ & $45 \%$ & $49 \%$ & $60 \%$ & $61 \%$ & $63 \%$ & $63 \%$ & $64 \%$ & $65 \%$ \\
$A U C^{d}$ & 0,533 & 0,573 & 0,595 & 0,648 & 0,643 & 0,638 & 0,613 & 0,610 & 0,608 \\
$J^{e}$ & 0,070 & 0,150 & 0,200 & 0,300 & 0,290 & 0,280 & 0,230 & 0,220 & 0,220 \\
\hline
\end{tabular}

Legenda: ${ }^{\text {V}}$ Valor preditivo positivo; ${ }^{\mathrm{b}}$ Valor preditivo negativo; ${ }^{\mathrm{c}}$ Precisão; ${ }^{\mathrm{d}}$ Área abaixo da curva de ROC; ${ }^{\mathrm{e}}$ Índice de Youden

O melhor ponto de corte que tem a sensibilidade mais elevada (78\%) é o 42,5 , com um valor de especificidade de $49 \%$ e um valor abaixo da curva ROC de 0,670 (IC 95\%=0,61-0,73). No entanto este ponto de corte, dados os valores apresentados pela escala, não é um valor com utilidade prática, considerando-se por este motivo o melhor ponto de corte o 45, por apresentar igual valor de sensibilidade (78\%) com um valor abaixo da curva ROC de 0,648
(IC 95\% =0,58-0,71) valor considerado razoável, um $J=0,30$ (IC 95\%=0,11-0,48) e uma especificidade aceitável de $52 \%$, (superior à do ponto de corte: $42,5)$ com um valor preditivo negativo de $82 \%$ e uma precisão igualmente aceitável de 60\% (Gráfico 1). Neste ponto de corte, $78 \%$ dos participantes são identificados como verdadeiros positivos e $22 \%$ como falsos negativos e apresenta um OR de 3,8 (IC $95 \%=2,17-6,51)$. 


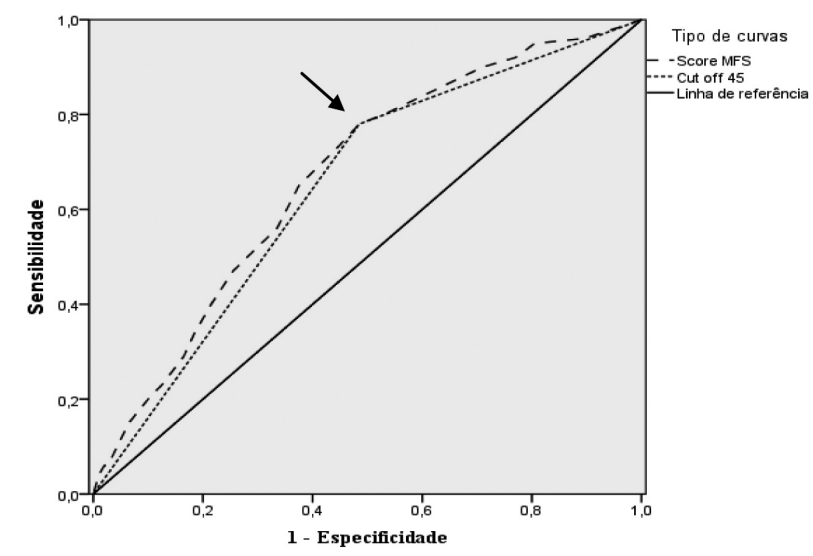

Legenda: Seta indica o pico mais elevado para o ponto de corte 42,5 e 45.

GRÁFICO 1 - Curva ROC com a relação entre sensibilidade e especificidade do ponto de corte do score da escala e do ponto de corte 45.

\section{Discussão}

A prevenção de quedas em pessoas adultas idosas é uma prioridade de saúde reconhecida. Neste estudo efetuou-se uma análise retrospetiva da sensibilidade e especificidade da MFS em doentes internados. A população alvo, para se definir o melhor ponto de corte da MFS, tinha uma idade com maior frequência absoluta e relativa de 75 anos, sendo o grupo etário entre os 70 e os 79 anos o que teve maior número de participantes e o que no qual ocorreram 34\% das quedas, resultados corroborados também já por estudos nacionais (Pina et al., 2010) e internacionais (Oliver et al., 2010). A média de idades dos participantes (76 anos) é superior à do estudo de Schwendimann et al. (2006), que foi de 70 anos.

Oprocessodeenvelhecimento, associadoàs alterações da capacidade física e cognitiva assim como a comorbilidade associada à doença crónica tem reflexos a nível hospitalar e muitos dos doentes internados no hospital são idosos e com vários problemas de saúde associados, tal como neste estudo em que $84 \%$ dos participantes têm um diagnóstico secundário.

Portugal enquadra-se hoje na realidade de outros países como a do Reino Unido, em que 60\% das admissões hospitalares são de pessoas com 65 ou mais anos e 70\% das camas estão ocupadas com pessoas desta faixa etária (Oliver, 2008), muitos destes doentes estão fragilizados pelo processo de doença e têm algum grau de défice cognitivo, tornando-os mais expostos à ocorrência de quedas. Os participantes deste estudo demonstram esse risco, dado que $40 \%$ destes, na avaliação feita pela MFS, se esqueciam das suas limitações e tinham, por esse motivo, 1,86 maior probabilidade de cair $(O R=1,86 ;$ IC $95 \%=1,14-3.03)$. Os homens caíram mais do que as mulheres, realidade já corroborada por outros estudos (Oliver et al., 2010). O maior número de quedas (42\%) registou-se no internamento de doentes com necessidade de convalescença e reabilitação, ou com doença crónica incurável, progressiva e avançada, efetivamente esta é uma das áreas de internamento já referida pelos autores como de maior risco (Morse, 2009; Oliver et al., 2010). No nosso estudo 52\% dos doentes internados neste serviço, apresentavam alto risco de queda, associado a 90\% um diagnóstico secundário, 51\% esquecem-se das suas limitações, 29\% terem história anterior de quedas, 33\% terem uma postura no andar debilitada e $25 \%$ dependente de ajuda, 21\% apoiam-se no mobiliário para andarem e 17\% utilizarem apoios de marcha.

O principal motivo de internamento de $36 \%$ dos participantes foi médico e quando comparados com os doentes internados por motivos cirúrgicos, os seus scores de risco são relativamente estáveis, melhorando ao longo do processo de internamento, sempre que a sua situação de saúde melhora também (Morse, 2009).

A doença oncológica foi o diagnóstico mais prevalente em $22 \%$ dos participantes. Os doentes com este diagnóstico caíram em média ao $10^{\circ}$ dia de internamento, mas a moda foi ao $2^{\circ}$ dia e $48 \%$ 
tinha alto risco de queda resultados corroborados por outros estudos (Stone, Lawlor, Savva, Bennett, \& Kenney, 2012). As quedas são mais comuns nos adultos com doença oncológica avançada, particularmente nos doentes com metástases cerebrais ou tumores primários do cérebro (Stone et al., 2012). Estudos retrospetivos em doentes internados com cancro avançado identificaram através de análise multivariada que a história anterior de quedas nos últimos 3 meses é um fator preditor de quedas neste tipo de pessoas (Stone et al., 2012), efetivamente 26\% das pessoas com doença oncológica tiveram quedas durante 0 seu internamento e na totalidade dos participantes estudados a história anterior de queda está associada a 2,19 maior probabilidade de cair $(O R=2,19$; IC $95 \%=1,25-3,83)$.

Utilizando pares de valores de sensibilidade e de especificidade para diversos pontos de corte optouse pelo ponto de corte 45 , embora utilizando os escores obtidos na avaliação efetuada da MFS fosse identificado como melhor ponto de corte o valor 42,5 . A razão desta opção tem a ver com o fato dos valores de pontuação da MFS serem zero, cinco ou múltiplos de cinco, sendo assim o valor 45 mais ajustado, valor igualmente sugerido pela autora (Morse, 2009) e por outros estudos (Chow et al., 2007).

Para além do fator anterior, importa também esclarecer que estes dois pontos de corte têm valores de sensibilidade iguais (78\%) e valores de especificidade idênticos (52\% vs. 49\%).

Em outros estudos efetuados em internamentos de Medicina foi identificado como melhor ponto de corte o 55 com uma sensibilidade de $75 \%$ e especificidade de 66\% (Schwendimann et al., 2006). Este ponto de corte é referido pela autora da escala como o valor máximo que o mesmo deve tomar (Morse, 2009).

No presente estudo o valor preditivo negativo foi de 82\% e no de Schwendimann et al. (2006) foi de 95\%, sendo assim melhor que o do nosso estudo. Ainda no estudo citado, a AUC foi de 0,701 e a ACC foi de $67 \%$ e no atual estudo foi de 0,648 e $60 \%$ respetivamente, de novo valores melhores que os por nós identificados.

No que se refere ao valor do Índice de Youden identificado neste estudo para o ponto de corte 45 , de 0,3 , foi o melhor encontrado em relação aos outros pontos de corte razão que também pesou na decisão final. Outros estudos identificaram valores mais baixos (Haines, Hill, Walshe, \& Osborne, 2007), fazendo com que a sua opinião sobre a utilização das escalas de avaliação do risco de queda e em especial da MFS fosse crítica.

Podemos assim recomendar que a que MFS e o ponto e corte 45 sejam utilizados em determinados contextos, idênticos aos do presente estudo, mas a utilização não deve ser generalizada, pois pode não trazer benefícios significativos para além de uma correta avaliação clínica do enfermeiro sobre o risco de queda do doente.

O foco dos enfermeiros deve ser colocado mais na prescrição das intervenções que visem determinar os meios apropriados para que o doente com risco beneficie dos mesmos.

Como limitações do estudo consideramos que ter-se efetuado um estudo de caso controlo com emparelhamento dos participantes por idade e sexo, pode ter condicionado que fossem encontradas associações entre o risco de queda e a idade ou com o sexo. O emparelhamento veio também dificultar encontrar para todos os casos os respetivos controlos e por isso perdemos alguns participantes, durante o ano em que decorreu o estudo. Mais difícil ainda, foi encontrar controlos para os participantes que caíram mais de uma vez, pois não foi possível selecionar controlos para estes participantes e o estudo não integrou assim pessoas com mais de uma queda.

\section{Conclusão}

As escalas de avaliação do risco de queda não devem ser utilizadas de forma acrítica e é necessário perceber as suas limitações e se descriminam suficientemente entre as pessoas com e sem risco de queda numa população específica. A sensibilidade, especificidade e os valores preditivos positivos e negativos, não são meros detalhes teóricos, tendo implicações reais na prática dos cuidados de enfermagem. Com os valores identificados neste estudo à semelhança de outros estudos não nacionais, podemos dizer que a MFS tem uma capacidade moderada de predizer o risco de queda, na tipologia dos internamentos estudados. Para os internamentos de adultos da área médico-cirúrgica e de cuidados continuados e paliativos recomenda-se a aplicação do ponto de corte 45, valor igualmente sugerido pela autora da escala. Recomenda-se que os pontos de corte da escala sejam estudados para cada realidade ou contexto. 


\section{Referências Bibliográficas}

Almeida, R. A., Abreu, C. C., \& Mendes, A. M. (2010). Quedas em doentes hospitalizados: Contributos para uma prática baseada na prevenção. Revista de Enfermagem Referência, 3 (2), 163-172. doi:10.12707/RIII1016.

Chow, S. K., Lai, C. K., Wong, T. K., Suen, L. K., Kong, S. K., Chan, C. K. , \& Wong, I. Y. (2007). Evaluation of the Morse Fall Scale: Applicability in Chinese hospital population. Internacional Journal of Nursing Studies, 44 (4), 556-565.

Eagle, D. J., Salama, S., Whitman, D., \& Evans, L. A. (1999). Comparison of three instruments in predicting accidental falls in selected inpatients in a general teaching hospital. Journal of Gerontological Nursing, 25 (7), 40-45.

HHaines, T. P., Hill, K., Walshe, W., \& Osborne, R. (2007). Designrelated bias in hospital fall risk screening tool predictive accuracy evaluations: Systematic review and meta analysis. The Journals of Gerontology. Series A, Biological Sciences and Medical Sciences, 62 (6), 664-672. Retirado de http:// hdl.handle.net/10536/DRO/DU:30019390.

Lamb, S. E., Jorstad-Stein, E. C., Hauer, K., \& Becker, C. (2005). Prevention of falls network Europe (PROFANE) outcomes consensus group. Development of a common outcome dataset for fall injury prevention trials. Journal of the American Geriatrics Society, 53 (9), 1618-1622. doi:10.1111/ j.1532-5415.2005.53455.x

Morse, J. (2006). The safety of safety research: The case of patient fall research. Canadian Journal of Nursing Research, 38 (2), 73-88.

Morse, J. (2009). Preventing patients falls: Establishing a fall intervention program ( $2^{\mathrm{a}}$ ed.). New York, NY: Springer Publishing Company.

Morse, J. M., Morse, R. M., \& Tylko, S. J. (1989). Development of a scale to identify the fall-prone patient. Canadian Journal on Aging, 8(4), 366-377.

Oliver, D. (2007). Preventing falls and fall injuries in hospitals: A major risk management challenge. Clinical Risk, 13 (5), 173178. doi:10.1258/135626207781572693
Oliver, D. (2008). "Acopia" and "social admission" are not diagnoses: Why older people deserve better. Journal of the Royal Society of Medicine, 101 (4), 168-174. doi:10.1258/ jrsm.2008.080017

Oliver, D., Daly, F., Martin, F., \& McMurdo, M. (2004). Risk factors and risk assessment tools for falls in hospital in-patients: A systematic review. Age and Ageing, 33 (2), 122-130. doi:10.1093/ageing/afh017

Oliver, D., Healey, F., \& Haines, T. P. (2010). Preventing falls and fall-related injuries in hospitals. In L. Z. Rubenstein \& D. A. Ganz, Clinics in geriatric medicine (Vol. 26, pp. 645-692). London, England: Elsevier.

Ordem dos Enfermeiros. (2004). Competências do enfermeiro de cuidados gerais. Lisboa, Portugal: Condelho de Enfermagem.

Perell, K. L., Nelson, A., Goldman, R. L., Prieto-Lewis, N., \& Rubenstein, L. Z. (2001). Fall risk assessment measures: An analytic review. Journal of Gerontology, 56 (12), 761-766. doi:10.1093/gerona/56.12.M761

Pina, S. M., Saraiva, D., Vaz, I., Ramalhinho, J., Ferreira, L., \& Batista, P. (2010). Quedas em meio hospitalar. Revista da Ordem dos Enfermeiros, 36, 27-29.

Schisterman, E. F., Perkins, N. J., Liu, A., \& Bondell, H. (2005). Optimal cut-point and its corresponding Youden Index to discriminate individuals using pooled blood samples. Epidemiology, 16 (1), 73-81. doi:10.1097/01. ede.0000147512.81966.ba

Schwendimann, R., De Geest, S., \& Milisen, K. (2006). Evaluation of the Morse Fall Scale in hospitalized patients. Age and Ageing, 35 (3), 311-313. doi:10.1093/ageing/afj066

Soares, M. E., \& Almeida, M. R. (2008). Acidentes com macas e camas em estabelecimentos bospitalares, envolvendo a

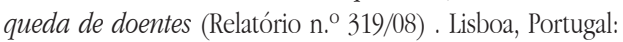
Inspecção Geral das Actividades em Saúde.

Stone, C. A., Lawlor, P. G., Savva, G. M., Bennett, K., \& Kenney, R. A. (2012). Prospective study of falls and risk factors for falls in adults with advanced cancer. Journal of Clinical Oncology, 30 (17), 2128-2133. doi:10.1200/JCO.2011.40.7791 